\title{
Hollow Core Photonic Bandgap fibers for Telecommunications: Opportunities and Potential Issues
}

\author{
Francesco Poletti, Eric Numkam Fokoua, Marco N. Petrovich, Natalie V. Wheeler, \\ Naveen Baddela, John R. Hayes, and David J. Richardson \\ Optoelectronics Research Centre, University of Southampton, Southampton SO17 1BJ, United Kingdom \\ frap@orc.soton.ac.uk
}

\begin{abstract}
We discuss the potential advantages that hollow core photonic bandgap fibers may ultimately have over conventional all solid fibers and review the state-of-the-art, fundamental physical limits and the many technological challenges to be overcome.

OCIS codes: (060.5295) Photonic crystal fibers; (060.2280) Fiber design and fabrication; (060.2330) Fiber optics communications
\end{abstract}

\section{Introduction}

The continuing exponential rise in demand for communications bandwidth provides a pressing need to find new solutions for increasing the overall capacity of optical fiber links. Coherent systems based on conventional fiber technology are already operating close to fundamental capacity and fiber loss limits. Consequently, radical new solutions including various forms of spatial division multiplexing (SDM) are currently under heavy investigation. The most radical solution of all is arguably that based on hollow core photonic bandgap fibers (PBGFs), which guide light in an air rather than a glass core [1]. As a result of this, PBGFs present two immediate advantages over all-solid fibers: a nearly 3 orders of magnitude lower effective nonlinearity and approximately $50 \%$ lower latency.

Although the debate is still ongoing as to the ultimate capacity and practical advantages offered by their extremely low nonlinearity $[2,3]$, there is no doubt that for PBGFs to ever be considered as a viable option for long haul data transmission, considerable improvements in some of their other optical properties will be needed. Although this is a challenging task, no fundamental physical limit is known which may ultimately prevent these fibers from achieving lower losses and wider bandwidth than conventional fibers. In this paper we will briefly review the state-of-the-art and challenges in the design, fabrication and use of PBGFs by focusing on 3 of the principal fiber properties: transmission bandwidth, loss and modal control.

\section{Bandwidth}

The effective useful bandwidth of a PBGF is a function of both the periodic cladding geometry and the core surround shape. The lattice arrangement and air-filling-fraction of the cladding specify the maximum theoretically available bandwidth of the fiber, while the core defect shape and most specifically the detail of how the photonic crystal is terminated around the core determine whether the full bandwidth can be exploited or whether surface modes (SMs) will in practice reduce the maximum useful bandwidth. The bandwidth to central wavelength ratio of a PBGF increases exponentially with the relative hole size $(d / \Lambda)$ and it typically ranges from 10-15\% in commercial fibers to $>30 \%$ in state-of-the-art fibers based on the standard triangular lattice of holes design. Considerably wider bandwidths, larger than $60 \%$, can theoretically be achieved by further increasing $d / \Lambda$ and employing square lattice (SL) or triangular lattice of rods (TLR) arrangements [4], see Fig. 1(a). This means that bandwidths approaching 100 $\mathrm{THz}$ are in principle available from such fibers.

However, it is well known that besides air-guided modes, PBGFs may also support SMs, guided in the glass core surround at wavelengths and propagation constants inside the bandgap. SMs can severely decrease the useful bandwidth of the fibers as a result of avoided crossing interactions with air guided modes, and also severely adversely affect their loss, birefringent properties and group delay difference (GDD) [5,6]. Since control of the spectral position of SMs is impossible in practice due to their extreme sensitivity to just nanometer scale variations in the glass core surround [7], SM elimination is a crucial step in the realization of PBGFs for telecoms applications.

\section{Loss}

Rayleigh scattering and infrared multiphonon absorption fundamentally limit the loss and define the optimum transmission window in conventional solid fibers. Since at telecoms wavelengths the Rayleigh contribution of air is negligible, loss in PBGFs is fundamentally dominated by a different physical phenomenon: surface scattering at the air-glass interfaces [8]. Surface scattering currently limits the lowest loss in a PBGF to above $1 \mathrm{~dB} / \mathrm{km}$ [8]. It is 
believed though that by adopting fiber designs that minimize the amount of electromagnetic field at the glass-air interfaces and glass processes that reduce the roughness of the inner surfaces after the fiber drawing process, significantly lower values of losses could in principle be achieved.

The simplest conceptual way to reduce the field intensity at the surfaces (often quantified by the F-parameter of the fiber [8]), is to enlarge the core radius $R$. Fig. 1(b) shows that F, to good approximation proportional to the scattering loss, scales roughly as $R^{-3}$. Since operating a PBGF at a different central wavelength $\lambda_{c}$ requires rigidly scaling all its structural dimensions, including $R$, such that the ratio $R / \lambda_{c}$ is conserved, the scattering loss component of a PBGF scales roughly as $\lambda_{c}^{-3}$ (as opposed to the typical Rayleigh $\lambda^{-4}$ dependence of conventional fibers). It is worth stressing though that such a spectral dependence arises predominantly from the structural scaling and is not a feature of the surface scattering itself, as is often erroneously believed. This can be observed in the simulations of surface scattering from a hollow tube using a modification of the dipole scattering method of Ref. [9], as shown in Fig. 1(c). These results can be extended to hollow core PBGFs and show that for a given fiber the wavelength dependence of the scattering loss within its spectral transmission region is much weaker than in conventional fibers. Therefore, if loss could be reduced to similar minimum levels, PBGFs would potentially offer much wider spectral regions of ultra-low loss transmission.

As a result of the small overlap of the optical modes with glass (typically $<0.1 \%$ ), the infrared glass absorption contribution to the overall loss is significantly reduced and the minimum loss region in PBGFs is shifted to longer wavelengths, approximately to between 1.9 and $2.0 \mu \mathrm{m}[8,10]$. Although operation at these wavelengths would require major changes in the network infrastructure, no fundamental road-blocks exist in semiconductor and active fiber technology to make such a change in wavelength an impossibility. Besides, operation around $1.9 \mu \mathrm{m}$ opens up the opportunity to exploit the very wide emission bandwidth of Tm, which at those wavelengths is capable of supporting a $\sim 20 \mathrm{THz}$ amplification bandwidth from a single amplifier, as opposed to the $15 \mathrm{THz}$ of the combined $\mathrm{S}+\mathrm{C}+\mathrm{L}$ regions, currently requiring 3 separate erbium-ion based amplifiers. This would represent an attractive step towards higher capacity optical networks with reduced complexity.

It is finally worth mentioning that the optimum operational wavelength range of PBGFs may ultimately not be determined by the spectroscopic properties of Tm, but rather by the desire to avoid the strongest roto-vibrational absorptions lines of water vapor or other gases such as $\mathrm{CO}_{2}$ or $\mathrm{HCl}$, which have been experimentally observed inside the fibers [10] and which may or may not be a reason of concern, depending on the minimum concentration levels ultimately achievable.

\section{Modal control}

As shown in Fig.1(b), although single mode PBGFs can be achieved with a small core made of only 3 missing elements [11], fibers with a much larger core will be in practice required to reduce the scattering loss to levels significantly below $1 \mathrm{~dB} / \mathrm{km}$. Since the number of guided modes in PBGFs scales roughly as $R^{2}$, low loss transmission fibers are expected to guide a multitude of modes. While in principle this offers the opportunity to enhance the transmission bandwidth by exploiting mode division multiplexing (MDM) schemes and transmitting separate information channels over separate modes, it also poses more stringent requirements on the modal characteristics of the fibers.

As already mentioned, SMs can drastically alter the dispersive properties of air-guided modes in an incontrollable way, and all efforts must be therefore devoted to their elimination. Some empirical design rules relying on the control of position and thickness of the core surround have been recently presented to achieve fibers where the fundamental mode is free of SM interactions [12,13]. For large core PBGFs, the core surround thickness obtained using the most conventional fabrication technique (which employs a larger central tube in the stacked preform to define the central core) does not conform to these rules. As a result, multiple undesired SMs are unavoidably supported, as shown in Fig. 1(d) for a 19 cell commercial fiber. These SMs will negatively affect the possibility of the fiber to operate efficiently in SDM schemes. However, using a modified fabrication approach which does not rely on the presence of the additional central capillary, it is possible to obtain large core fibers with a thin enough core boundary to allow them to operate virtually SM-free, as shown in Fig. 1(e).

Once SMs are properly eliminated for all modes, the most appropriate MDM scheme to be used with PBGFs will ultimately depend on the amount of GDD and intermodal cross-coupling introduced. In principle, to limit the complexity and size of digital signal processing circuits in MIMO transmissions, fibers with as low as possible a GDD between all the modes of interest are desirable. As shown in Fig. 1(f) the simulated GDD of an ideal 19 cell core fiber with $R=11 \mu \mathrm{m}$ is larger than for low-GDD multimode solid counterparts. Significantly lower values of GDD in PBGFs can be obtained by further enlarging the core radius. Additional work to experimentally quantify the intermodal crosstalk or multipath interference introduced by PBGFs is required at this stage. 
(a)

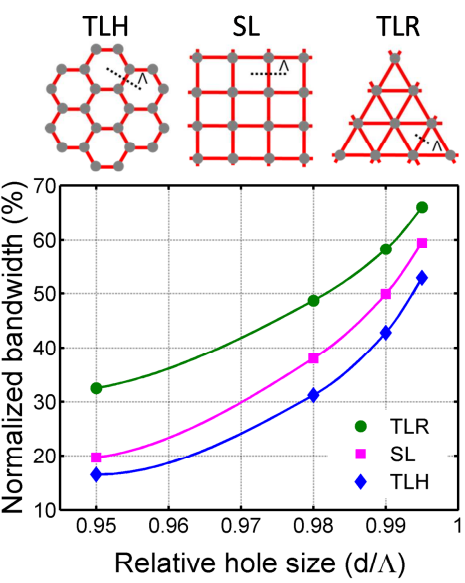

(d)

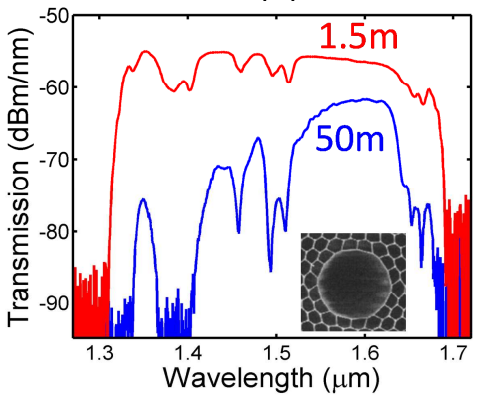

(b)
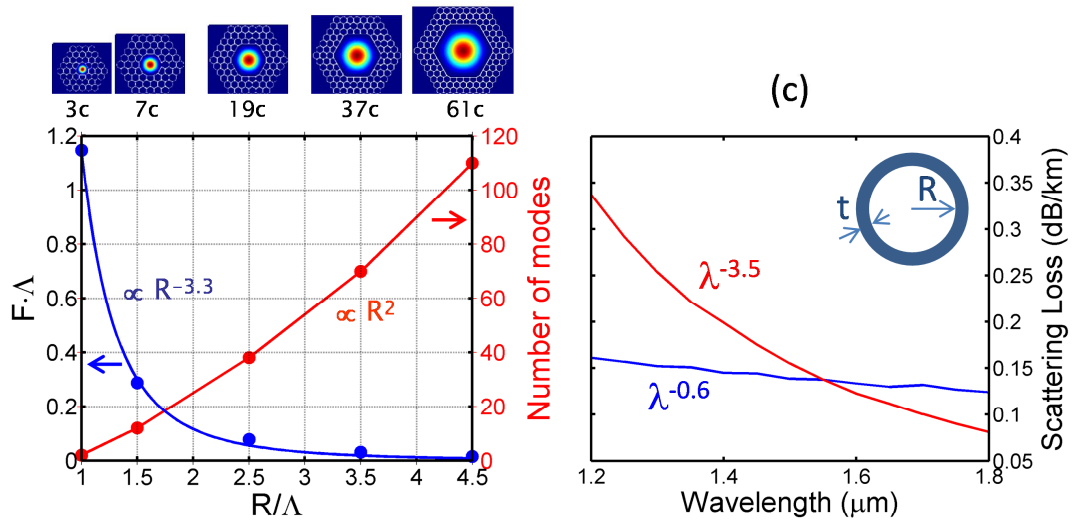

(e)

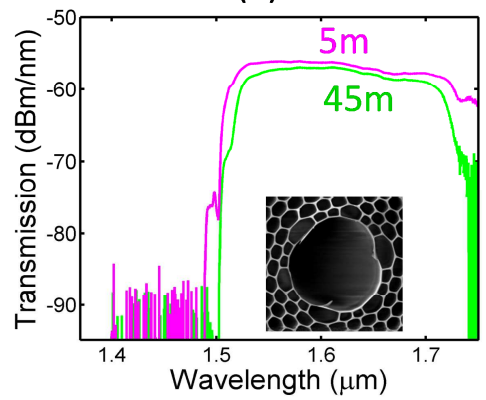

(f)

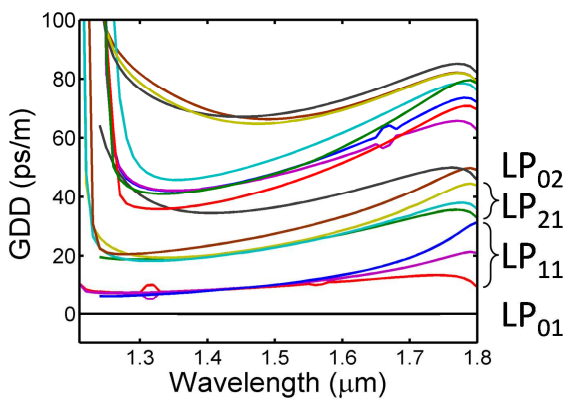

Fig.1: (a) Maximum normalized bandwidth vs $d / \Lambda$ for 3 different lattice arrangements; (b) dependence of F-parameter and number of air guided modes from the core radius of an ideal PBGF; (c) wavelength dependence of the scattering loss from a hollow cylinder where the only thickness $t$ is adjusted to maintain antiresonance (blue) and where also the radius $R$ is changed such that $R / \lambda$ is constant (red); (d) transmission over short and long length of a commercial 19 cell PBGF with thick core surround; (e) transmission of a custom made thin core 19 cell PBGF showing surface mode-free operation; (f) Simulated GDD of the main air guided modes of a 19 cell core $\operatorname{PBGF}(R=11 \mu \mathrm{m})$ with respect to the fundamental mode.

\section{Conclusions}

We have reviewed the state-of-the art in PBGF technology and highlighted the potential advantages these fibers may offer in terms of bandwidth and nonlinearity. We have stressed the deleterious role of surface modes on almost all the fiber's optical properties and shown good progress towards their elimination.

This work was supported by the Royal Society and by the EU FP7-ICT under grant 258033 (MODE-GAP).

\section{References}

[1] R. F. Cregan, et al., "Single-mode photonic band gap guidance of light in air," Science 285, 1537-1539 (1999).

[2] A. D. Ellis et al., "Capacity in Fiber Optic Communications- The Case for a Radically New Fiber," IPC 2011 , paper TuN1.

[3] R. J. Essiambre, "Impact of Fiber Parameters on Nonlinear Fiber Capacity," OFC 2011, paper OTuJ1,

[4] F. Poletti, "Hollow core fiber with an octave spanning bandgap," Opt Lett 35, 2837-2839 (2010).

[5] F. Poletti et al., "The effect of core asymmetries on the polarization properties of hollow core photonic band gap fibers," Opt Express 13, 9115-9124 (2005)

[6] J. A. West, et al., "Surface modes in air-core photonic band-gap fibers," Opt Express 12, 1485-1496 (2004).

[7] F. Poletti et al., "Advances and limitations in the modeling of fabricated photonic bandgap fibers," OFC 2006 , paper OFC2.

[8] P. J. Roberts et al., "Ultimate low loss of hollow-core photonic crystal fibres," Opt Express 13, 236-244 (2005).

[9] E. G. Rawson, "Analysis of Scattering from Fiber Waveguides with Irregular Core Surfaces," Appl Optics 13, 2370-2377 (1974).

[10] J. K. Lyngso et al., "7-cell core hollow-core photonic crystal fibers with low loss in the spectral region around $2 \mu \mathrm{m}, "$ Opt Express 17 , 23468-23473 (2009).

[11] M. N. Petrovich et al., "Robustly single mode hollow core photonic bandgap fiber," Opt Express 16, 4337-4346 (2008).

[12] H. K. Kim et al., "Designing air-core photonic-bandgap fibers free of surface modes," IEEE J Quantum Elect 40, 551-556 (2004);

[13] R. Amezcua-Correa et al., "Design of 7 and 19 cells core air-guiding photonic crystal fibers for low-loss, wide bandwidth and dispersion controlled operation," Opt Express 15, 17577-17586 (2007); 